\title{
Giant permanent dipole moments of excitons in semiconductor nanostructures
}

\author{
R. J. Warburton, ${ }^{1}$ C. Schulhauser, ${ }^{2}$ D. Haft, ${ }^{2}$ C. Schäflein, ${ }^{2}$ K. Karrai, ${ }^{2}$ J. M. Garcia, ${ }^{3}$ W. Schoenfeld, ${ }^{4}$ and P. M. Petroff ${ }^{4}$ \\ ${ }^{1}$ Department of Physics, Heriot-Watt University, Edinburgh EH14 4AS, United Kingdom \\ ${ }^{2}$ Center for NanoScience and Sektion Physik, Ludwig-Maximilians-Universität, Geschwister-Scholl-Platz 1, \\ D-80539 München, Germany \\ ${ }^{3}$ Instituto de Microelectronica de Madrid, CNM-CSIC Isaac Newton, 8, PTM, 28760 Madrid, Spain \\ ${ }^{4}$ Materials Department and QUEST, University of California, Santa Barbara, California 93106
}

(Received 20 June 2001; published 15 February 2002)

\begin{abstract}
We have measured the vertical Stark effect of excitons confined to individual self-assembled ring-shaped quantum dots. We find that the excitons have very large permanent dipole moments corresponding to electronhole separations up to $2.5 \mathrm{~nm}$, comparable to the nanostructures' physical height. We find a trend of both permanent dipole moment and polarizability on the emission energy, but a very strong correlation between the permanent dipole moment and the polarizability.
\end{abstract}

DOI: 10.1103/PhysRevB.65.113303

PACS number(s): 78.67.Bf, 73.21.-b, 73.40.Rw, 78.66.Fd

Semiconductor quantum dots combine two contemporary themes: they are nanometre-sized and they can be selfassembled. They confine electrons and holes in all three directions, and this property is making them extremely attractive for photonics devices, not just for improved laser diodes, ${ }^{1}$ but also for single photon sources ${ }^{2}$ and detectors. ${ }^{3}$ There are many open questions concerning the strain and composition of quantum dots and how structural properties relate to electronic properties. Furthermore, the extent to which the electronic properties of quantum dots can be tailored is not yet known.

In the vertical direction, there are different confining potentials for electrons and holes leading to a spatial separation of the electron and hole wave functions, i.e., a permanent dipole moment. In addition, the exciton has a polarizability, the extent to which an applied electric field can pull the electron and hole apart, reducing the energy. Both parameters are important. The permanent dipole moment influences the excitonic oscillator strength and in addition, it is sensitive to the detailed structure of the dots. The polarizability determines the sensitivity of the exciton energy to an electric field. For applications of quantum dots as emitters and detectors, the polarizability should be large so that the energy can be tuned over a large range with modest electric fields. Conversely, for applications involving coherent control or high-resolution spectroscopy, the polarizability should be small because the Stark effect contributes to the line width, in particular of single dot emission, through the local electric fields generated by fluctuating charge.

InAs quantum dots in GaAs have an electron-hole separation of about $0.4 \mathrm{~nm}$, with the hole localized at the apex of the dot, above the electron whose wave function is spread over the entire dot. ${ }^{4}$ The sign of this dipole moment has been interpreted as, first, evidence of a strong indium concentration gradient, with the apex indium-rich and the base indiumpoor, and, second, of a truncation in the capped dots relative to uncapped dots, ${ }^{4}$ conclusions which have now been confirmed by structural studies. ${ }^{5,6} \mathrm{We}$ present here measurements showing how we are able to tune the excitonic perma- nent dipole moment and polarizability of quantum dots by adjusting the self-assembly procedure.

Our experiments use quantum rings embedded in the insulator region of a metal-insulator-semiconductor heterostructure with which we have already demonstrated single electron charging of a single quantum ring. ${ }^{7}$ The quantum rings are situated $25 \mathrm{~nm}$ above an $n^{+}$region, and $150 \mathrm{~nm}$ below the surface. We evaporate a semi-transparent gate electrode on to the surface and apply a voltage $V_{g}$ between gate and back contact. The quantum rings are grown by first depositing InAs on GaAs in the Stranski-Krastanow growth mode, producing lens-shaped quantum dots, $20 \mathrm{~nm}$ in diameter, $6 \mathrm{~nm}$ high, with a density in the $10^{9} \mathrm{~cm}^{-2}$ range. We then deposit $1 \mathrm{~nm}$ of GaAs, after which we anneal at the growth temperature of $530{ }^{\circ} \mathrm{C}$ for about $30 \mathrm{~s}$. During this annealing step, there is a significant movement of material laterally with a reduction in height to $2 \mathrm{~nm}$. The astonishing feature is the appearance of a crater in the center of the dots, generating the ringlike structure. ${ }^{8,9}$ The radius at maximum height is $\sim 18 \mathrm{~nm}$. After the annealing step, the capping is completed by depositing more GaAs. The rings have a multipeaked distribution, giving rise to a weak emission band at $1.27 \mathrm{eV}$ and two strong emission bands at 1.30 and $1.34 \mathrm{eV}$ in the ensemble photoluminescence. We have measured the photoluminescence (PL) of individual rings as a function of $V_{g}$ at $4.2 \mathrm{~K}$ with a confocal microscope, using $300 \mathrm{~nm}$ sized holes in an opaque metal mask to resolve single rings.

Typical behavior is shown in Fig. 1. For $V_{g}<-0.4 \mathrm{~V}$, the PL is quenched because the electron tunneling time is smaller than the excitonic radiative lifetime. ${ }^{7}$ The single line for $V_{g} \sim-0.3 \mathrm{~V}$ is the PL from neutral excitons $\left(X^{0}\right)$. At $V_{g}=-0.24 \mathrm{~V}$, the emission redshifts due to the formation of charged excitons $\left(X^{1-}\right)$. We focus here on the Stark effect, the change in PL energy with vertical electric field. For each ring, we plot the PL energy as a function of $V_{g}$ with the abrupt shifts in the PL energy subtracted. We find that the PL energy can always be fitted accurately to a quadratic function of $V_{g}$, exactly as expected for a polarizable dipole in an electric field. Two examples are shown in Fig. 2. We note that the Stark shift is not appreciably modified by any excess 


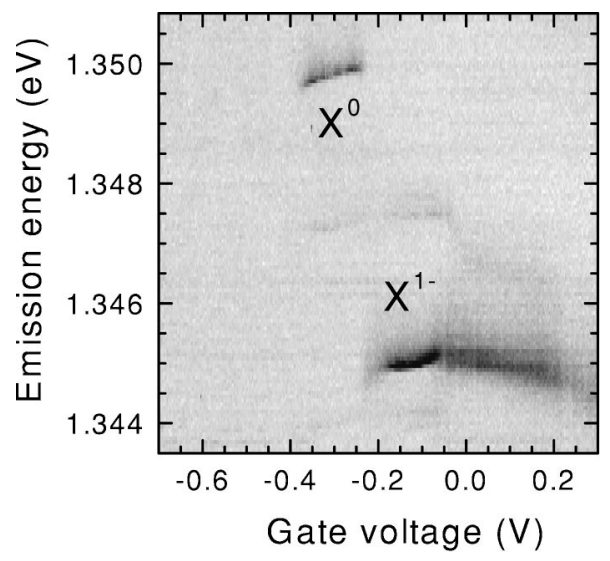

FIG. 1. A gray-scale plot of the photoluminescence (PL) of a single quantum ring versus gate bias $V_{g}$.

charge because the Stark shift of the Coulomb energies is small compared to the Stark shift of the electron and hole. ${ }^{10}$

We describe an exciton in each ring with a permanent dipole moment $p$ and a polarizability $\beta$, so that the PL energy $E_{\mathrm{PL}}$ is given by $E_{\mathrm{PL}}=E_{o}-p F+\beta F^{2}$, where $F$ is the electric field. $F$ is linearly related to the gate voltage $V_{g}: F$ $=-\left(V_{g}-V_{g}^{0}\right) / d$, where $V_{g}^{0}$ is the Schottky barrier potential and $d$ is the distance between the $n^{+}$layer and the sample surface. We employ the sign convention that a positive $F$ points from the wetting layer towards the sample surface. ${ }^{4}$ We have measured $V_{g}^{0}$ for our structure from the photocurrent induced by light with above GaAs-band gap energy. The signal changes sign for flat bands, determining $V_{g}^{0}=0.62 \mathrm{~V}$. We take $d$ to be $175 \mathrm{~nm}$, the target value from the growth. We have confirmed this value by measuring the capacitance between a known gate area and the back contact. Overall, we can determine $F$ from $V_{g}$ to better than $10 \%$ accuracy.

We have determined $p$ and $\beta$ for more than 20 individual rings. We express $p=e r$ where $e$ is the electronic charge and $r$ is the separation between the center-of-gravity of the electron and hole wave functions in the growth direction. In Fig.

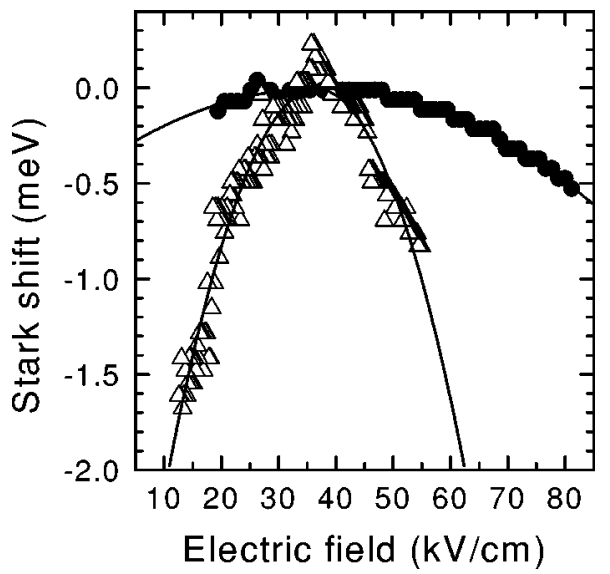

FIG. 2. The shift in PL energy versus electric field for two individual quantum rings, one weakly polarizable $\left(E_{0}=1.266 \mathrm{eV}\right.$, solid circles) and one highly polarizable $\left(E_{0}=1.363 \mathrm{eV}\right.$, open triangles). The solid curves are quadratic fits to the data.

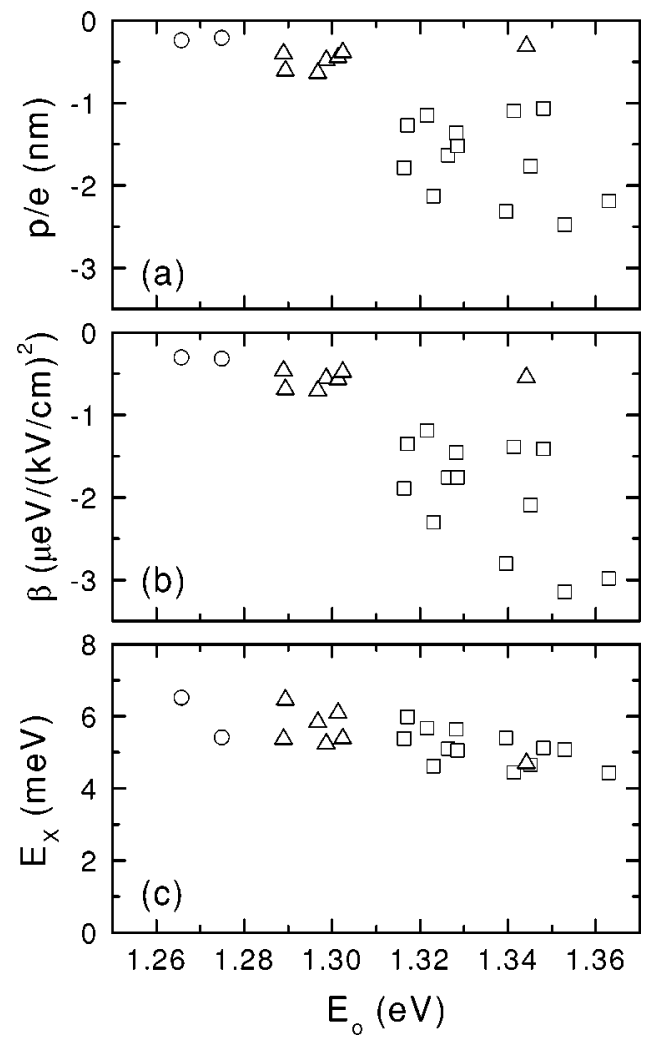

FIG. 3. (a) The permanent dipole moment $p$, (b) the polarizability $\beta$, and (c) the energetic difference between the neutral and singly charged exciton $E_{X}$, plotted against $E_{0}$, the energy of the neutral exciton at zero electric field. Each point was recorded on an individual quantum ring. The symbols identify to which ensemble peak each ring belongs. Circle, triangle, square: ensemble PL peaked at $1.27,1.30,1.34 \mathrm{eV}$, respectively.

3 , we plot $r$ and $\beta$ against $E_{0}$, each point representing the result for one ring. Both $r$ and $\beta$ increase in magnitude with increasing $E_{0}$, but with large fluctuations from ring to ring. There are two surprising features. First, the permanent dipole moments are large. The average value is $\bar{r}=-1.2 \mathrm{~nm}$ [standard deviation $\sigma(r)=0.7 \mathrm{~nm}$ ], with some of the rings having dipole moments as large as $-2.5 \mathrm{~nm}$. These values are both opposite in sign and much larger in magnitude than those previously reported $\left[r=0.4 \mathrm{~nm},{ }^{4} r=0.62 \mathrm{~nm},{ }^{11} r\right.$ $=0.5 \mathrm{~nm}$ (Ref. 12)]. Secondly, there is a clear linear relationship between $r$ and $\beta$, as shown in Fig. 4 .

The permanent dipole moment is very sensitive to the details of the vertical electron and hole confining potentials. ${ }^{13}$ Calculations assuming pure InAs dots predict negative dipole moments, with the hole confined at the base of the dot, below the electron. ${ }^{14}$ Conversely, calculations assuming an indium-rich apex tend to predict positive dipole moments. This is because the indium-rich apex always has the largest uniaxial strain which has a powerful confining effect on the holes. The electrons are unaffected by the uniaxial strain, and they are lighter than the holes, enabling the electron wave function to delocalize over the dot. For uncapped dots grown in our chamber, we have very strong evidence from $\mathrm{x}$-ray measurements for an indium-rich top and indium-poor base ${ }^{6}$ which in the light of all recent 


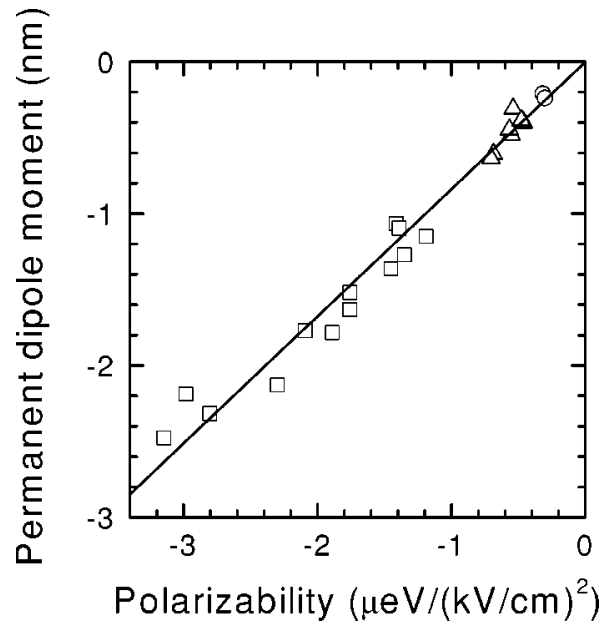

FIG. 4. The permanent dipole moment against the polarizability for 22 quantum rings. The solid line is a linear fit.

work $^{4,11-13}$ must have positive dipole moments. The reversed dipole moment of the quantum rings is therefore a consequence of the annealing step. The annealing process induces a reduction in height from 6 to $2 \mathrm{~nm}$. However, in order to account for the negative dipole moments of the excited quantum rings, there must be a movement of indium vertically, into the nanostructures. On deposition of the cap GaAs material, the indium-rich apex of the nanostructures is destabilized through a reduction in the misfit strain energy, probably giving rise to a dissolution of the apex during the anneal. This has been observed directly with the capping of SiGe nanostructures with $\mathrm{Si}^{15}$ in the present case, we postulate that indium plays the same role as $\mathrm{Ge}$ in the $\mathrm{Si} / \mathrm{Ge}$ system. Indium migration is also consistent with the formation of the pronounced crater in the middle of the structures; during the annealing, there is a pronounced movement of indium away from the central apex both downwards and outwards.

To interpret the results on the polarizability, we assume a parabolic confinement in the vertical direction $\mathbf{z}$, so that the Hamiltonian for an electron is $H=\frac{1}{2} m_{e} \omega_{e, z}^{2} z^{2}-e F z$, where $\hbar \omega_{e, z}$ is the quantization energy, and $m_{e}$ the effective mass. The Stark shift of all states is $\Delta E=-\left(e^{2} m_{e} L_{e, z}^{4} / 2 \hbar^{2}\right) F^{2}$, where $L_{e, z}$ is the extent of the electron ground state wave function in $\mathbf{z}, L_{e, z}=\sqrt{\hbar / m_{e} \omega_{e, z}}$, with similar results pertaining to the hole. This result encapsulates a general point: the polarizability depends on the mass and strongly on the spatial extent of the carrier. Electrons and holes contribute roughly equally to the polarizability because although the hole has a much larger mass than the electron, it has a slightly smaller extent. The average value of the polarizability we measure $\left[\bar{\beta}=-1.4, \sigma(\beta)=0.9 \mu \mathrm{eV} /(\mathrm{kV} / \mathrm{cm})^{2}\right]$ gives quantization energies (vertical extents) of about 95 $\mathrm{meV}(3.4 \mathrm{~nm})$ and $40 \mathrm{meV}(2.6 \mathrm{~nm})$ for the electrons and holes, respectively, assuming effective masses of 0.07 and 0.3 and that $L_{e, z} / L_{h, z} \sim 1.3$, as for the lateral confinement of our InAs dots. ${ }^{16}$ The polarizability tends to increase with increasing PL energy [Fig. 3(b)]. The effective masses will not change much from ring to ring, implying that the confinement lengths tend to increase with increasing PL energy. In other words, the potential becomes slightly softer. How- ever, the Stark effect has such a strong dependence on $L_{e, z}$ and $L_{h, z}$ that only a standard deviation of $\sim 20 \%$ in the distribution of $L_{e, z}$ and $L_{h, z}$ is required to account for the variation in $\beta$ we see experimentally.

To explore the relationship between the permanent dipole moment and the polarizability of the excitons, we pursue the argument with the harmonic confining potential, introducing a permanent dipole moment through an in-built electric field $F_{0}$. The total electric field becomes $F+F_{0}$. It is simple to show that $p / \beta=-2 F_{0}$. This means that a constant ratio of $p$ to $\beta$ is equivalent to a constant in-built electric field. Experimentally, we find that the average in-built field is $\bar{F}_{0}$ $=-42 \mathrm{kV} / \mathrm{cm}$, with small fluctuations from ring to ring, $\sigma\left(F_{0}\right)=5 \mathrm{kV} / \mathrm{cm}$. We can rule out an unusual pinning of the Fermi energy at the surface as an explanation for this almost ring-independent $F_{0}$ through our measurement of $V_{g}^{0}$. A piezoelectric field, of negligible importance for the ground state exciton of a quantum dot, ${ }^{13,14}$ may be important for quantum rings, but it strikes us as unlikely that the piezoelectric field is the same for all rings. We gain some insight into this problem from a classical interpretation by imagining the electron and hole wave functions to represent the two plates ( $\operatorname{area} A$ ) of a capacitor. The electric field between the plates is $F_{0}=e / A \varepsilon_{0} \varepsilon_{r}$, independent of the plate separation, a consequence of Gauss' Law. The conclusion is that a constant $F_{0}$ arises because the lateral area $A$ of the exciton varies little from ring to ring.

A constant lateral excitonic size is consistent with the fact that the energy shift between neutral and singly charged excitons, $E_{X}$, is almost independent of $E_{0}$ [Fig. 3(c)]. The shift arises because in the plane, $L_{e}>L_{h}$, and it scales as $1 / L_{e}$ for constant $L_{e} / L_{h} \cdot{ }^{16}$ The slight decrease in $E_{X}$ with $E_{0}$ implies a slight softening in the potential, and correspondingly, for increasing $E_{0}$ we find a slight decrease in $F_{0}$ (increase in $A$ ) within each group of ring.

The measured $F_{0}$ implies a lateral excitonic area of $360 \mathrm{~nm}^{2}$. According to capacitance spectroscopy, ${ }^{9}$ the electronic radius is $14 \mathrm{~nm}$. Taking $14 \mathrm{~nm}$ also for the excitonic radius, our measured area implies a ring width of $4 \mathrm{~nm}$. We note that both the radius and width are larger than the maximum value of $r$, as required for the consistency of our analysis, but also that the details of the lateral excitonic confinement are not well understood at present.

In the light of this analysis, we predict a linear relationship between the excitonic permanent dipole moment and polarizability for other Stranski-Krastanow systems as it depends only on a roughly constant lateral extent, and a much smaller vertical extent, which are likely to be general features. In contrast, CdSe nanocrystals do not show the same relationship between $p$ and $\beta$ although the magnitudes of $p$ and $\beta$ are similar to the results reported here. ${ }^{17}$ One reason is that the CdSe nanocrystals have hard confining potentials.

In conclusion, we have used the Stark effect to explore the vertical confinement of excitons in ringlike quantum dots. The excitons exhibit large and negative permanent dipole moments with the separation of the electron and hole wave functions comparable to the physical thickness. We argue 
from this that the indium profile of the rings is radically altered by our capping procedure. The polarizability increases with increasing emission energy which we interpret as a softening in the vertical potential. Finally, for a wide variety of rings, we uncover a linear relationship between the permanent dipole moment and the polarizability which we argue is a consequence of an almost ring-independent lateral extent. These results open up the possibility of tailoring the polarizability for particular applications.

We would like to thank Kevin A. Prior and David E. Jesson for stimulating discussions. This work was funded by the Deutsche Forschungsgemeinschaft (Grant No. SFB 348) and EPSRC of the UK.
${ }^{1}$ See, for example, D. Bimberg, M. Grundmann, and N. N. Ledentsov, Quantum Dot Heterostructures (Wiley, New York, 1998).

${ }^{2}$ P. Michler et al., Science 290, 2282 (2000).

${ }^{3}$ A.J. Shields et al., Appl. Phys. Lett. 76, 3673 (2000).

${ }^{4}$ P.W. Fry et al., Phys. Rev. Lett. 84, 733 (2000).

${ }^{5}$ N. Liu, J. Tersoff, O. Baklenov, A.L. Holmes, Jr., and C.K. Shih, Phys. Rev. Lett. 84, 334 (2000).

${ }^{6}$ I. Kegel et al., Phys. Rev. Lett. 85, 1694 (2000).

${ }^{7}$ R.J. Warburton et al., Nature (London) 405, 926 (2000).

${ }^{8}$ J.M. Garcia et al., Appl. Phys. Lett. 71, 2014 (1997).

${ }^{9}$ A. Lorke et al., Phys. Rev. Lett. 84, 2223 (2000).

${ }^{10}$ This viewpoint is familiar from quantum well physics. See for example G. Bastard, Wave Mechanics Applied to Semiconductor Heterostructures (Wiley, New York, 1988).

${ }^{11}$ T.M. Hsu, W.-H. Chang, C.C. Huang, N.T. Yeh, and J.-I. Chyi, Appl. Phys. Lett. 78, 1760 (2001).

${ }^{12}$ F. Findeis, M. Baier, E. Beham, A. Zrenner, and G. Abstreiter, Appl. Phys. Lett. 78, 2958 (2001).

${ }^{13}$ J.A. Barker and E.P. O'Reilly, Phys. Rev. B 61, 13840 (2000).

${ }^{14}$ M. Grundmann, O. Stier, and D. Bimberg, Phys. Rev. B 52, 11969 (1995); C. Pryor, ibid. 57, 7190 (1998); J. Kim, L.-W. Wang, and A. Zunger, ibid. 57, R9408 (1998).

${ }^{15}$ P. Sutter and M.G. Lagally, Phys. Rev. Lett. 81, 3471 (1998).

${ }^{16}$ R.J. Warburton et al., Phys. Rev. B 58, 16221 (1998).

${ }^{17}$ S.A. Empedocles and M.G. Bawendi, Science 278, 2114 (1997). 